Information from the American Diabetes Association for people with diabetes

GOOD TO KNOW

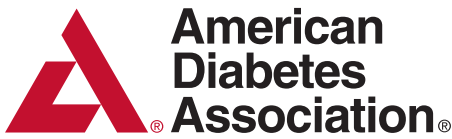

\title{
All About Insulin Resistance
}

Insulin resistance is a condition that raises your risk for type 2 diabetes and heart disease. When you have insulin resistance, your body has problems using insulin. Over time, this makes your blood glucose (sugar) levels go up. The good news is that being active and losing weight can reverse insulin resistance and lower your risks.

\section{What does insulin do?}

Insulin helps your body use glucose for energy. Your body breaks food down into glucose and sends it into the blood. Insulin helps move the glucose from the blood into your cells.

When you have insulin resistance, your body can't use insulin properly. At first, your pancreas responds by making extra insulin. But, over time, it can't make enough insulin to keep your blood glucose at normal levels. If your blood glucose gets too high, you may have either prediabetes or diabetes.

\section{How is insulin resistance diagnosed?}

People with insulin resistance usually have no symptoms. Your health care provider can review your risk factors. If you're at risk, your health care provider should check your blood glucose levels to see whether you might have prediabetes or diabetes.

\section{Does insulin resistance affect people who already have} type 2 diabetes?

Yes, insulin resistance is why insulin isn't used properly in people with type 2 diabetes. Diabetes is a progressive disease-even if you don't need to treat it with medications at first, you may need to later. If you have type 2 diabetes, control your blood glucose by eating fewer calories, being more active, and losing excess weight. Talk with your health care team about ways to achieve your goals using meal planning, physical activity, and medicine.

\section{WHAT RAISES YOUR RISK FOR INSULIN RESISTANCE}

You are at risk if you:

- are overweight

- are physically inactive

- have a parent, brother, or sister with type 2 diabetes

- are African American, Hispanic/Latino, American Indian, Asian American, or Pacific Islander

- have polycystic ovary syndrome, also called PCOS

- have had gestational diabetes, which is diabetes that develops during pregnancy

- have given birth to a baby weighing more than 9 pounds

- are age $\mathbf{4 5}$ or older

- have had above-normal blood glucose levels

- have high blood pressure

- have low HDL (good) cholesterol

- have high levels of blood fats called triglycerides

- have had heart disease, a stroke, or disease of the blood vessels in your neck or legs

Some of these risk factors also raise your risk for heart disease. 
HOW YOU CAN PREVENT OR REVERSE INSULIN RESISTANCE
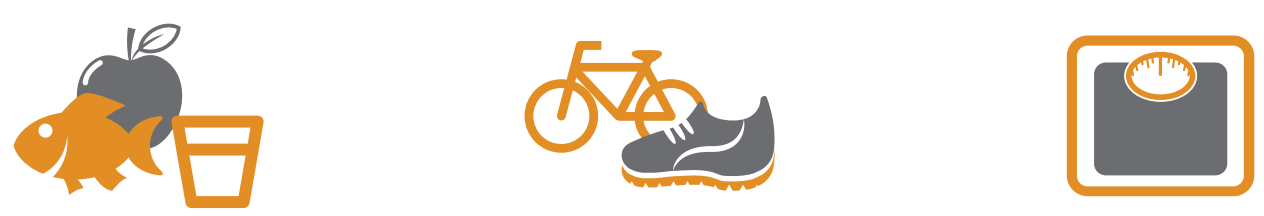

You can eat fewer calories and be physically active, which will help you lose excess weight. Studies have shown that losing even $7 \%$ of your weight may help. For example, if you weigh 200 pounds, your goal would be to lose 14 pounds.

\section{Eat less}

Place a check mark next to the steps you'd like to try to eat fewer calories. A dietitian can help you find other ways to eat less and plan healthy meals.

\section{Eat smaller serving sizes.}

Order the smallest serving size when eating outor share your main dish. You also can divide your meal and take half home for the next day.

$\square$ Try calorie-free drinks or water instead of regular soft drinks and juice.

$\square$ Choose baked, grilled, and steamed foods instead of fried.

\section{$\square$ Use a smaller plate.}

$\square$ Fill half your plate with greens and veggies. Fill $1 / 4$ with meat or other protein, and $1 / 4$ with carbs, such as brown rice or whole-grain pasta.

Eat more vegetables, whole grains, and fruit.

Ask for salad dressings and sauces on the side and then use as little as possible.

$\checkmark$ Use nonstick pans or cooking sprays.

$\square$ Cut back on high-fat toppings, such as butter, margarine, sour cream, regular salad dressing, mayonnaise, and gravy. Instead, season foods with barbecue sauce, salsa, lemon juice, or other low-fat options.

Eat small servings of low-calorie, low-fat snacks.

Another way l'll try to eat fewer calories is to:

\section{Be physically activity}

Place a check mark next to the ways you'll try to add physical activity to your routine.

$\square$ Get up and move every 90 minutes if you sit for long periods of time.

$\square$ Take the stairs instead of the elevator.

$\square$ Walk around while you talk on the phone or during TV commercials.

$\square$ Find an activity you enjoy, such as dancing, gardening, or playing with the kids. Move more around the house. For example, clean the house, work in the garden, or wash the car.

$\square$ Take the dog for a walk.

$\square$ Park at the far end of the parking lot and walk to the store.

$\square$ Walk every day, working up to 30 minutes of brisk walking, 5 days a week-or split the 30 minutes into two 15-minute or three 10 -minute walks.

Try strength training by lifting light weights two to three times a week.

$\square$ Another way l'll try to add physical activity to my day is to:

This hand-out was published in Clinical Diabetes, Vol. 36, issue 3, 2018, and was adapted from the American Diabetes Association's Cardiometabolic Toolkit No. 2: All About Insulin Resistance. Visit the Association's Patient Education Library at http://professional.diabetes.org/PatientEd for hundreds of free, downloadable handouts in English and Spanish. Distribute these to your patients and share them with others on your health care team. Copyright American Diabetes Association, Inc., 2018. 